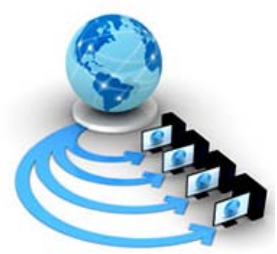

\title{
UPPER BOUNDS OF DIFFERENT TRAFFIC TYPES IN IEEE 802.22 BASED CRNS
}

\author{
Nikita Bhagat \\ CSE Department \\ GNDU, RC, JALANDHAR
}

\author{
Dr. Jyoteesh Malhotra \\ ECE Department \\ GNDU, RC, JALANDHAR
}

\begin{abstract}
Data communication networks are a vital component of any modern society. They are used extensively in numerous applications, including financial transactions, social interactions, education, national security, and commerce. In particular, both wired and wireless devices are capable of performing a plethora of advanced functions that support a range of services, such as voice telephony, web browsing, streaming multimedia, and data transfer [1]. With the rapid evolution of microelectronics, wireless transceivers are becoming more versatile, powerful, and portable. In the wireless technology the major issue is the issue of spectrum utilization. The utilization of spectrum has increased to its maximum level. To tackle this issue the technology named cognitive radio was introduced to the rescue. The cognitive radio is an emerging technology that facilitate dynamic spectrum access in wireless networks. The cognitive radio is capable of expediently using the obtainable portions of a licensed spectrum to improve the application performance for unlicensed users. The opportunistic access of the accessible channels in the wireless environment requires dynamic channel assignment to efficiently utilize the available resources while minimizing the interference in the network. In this paper we are focusing the different traffic types such video and voice. In the voice we have different codec and in the video application we have different model types from which we need to choose the best for the optimum results.
\end{abstract}

Keywords: Cognitive radio, IEEE 802.22, NETSIM, video and voice application, codec, model type.

\section{INTRODUCTION}

In this modern era of science and technology, where every person is aware of the latest technology and trends, every second person is holding mobile phone and using its resources in the well manner, this leads to the situation where a person need full access of the resources for which him/her paying. Now the situation has arisen where the efficient utilization of resources is required. The craze of internet has increased as the smallest to the biggest thing is available on the internet, solution to every knowledge based problem is present on the internet.

At present, the information and communication technology commerce is facing global provocation in developing new services and products with enhanced Quality of Service (QoS). Undoubtedly, there is a keen call for global coherence not only in terms of energy, but also in terms of spectra available. In fact, the gap between supply and demand in wireless domain is increasing. With the shatter demand from smart phones to run bigger number of applications with paramount data rates will soon engulf the wireless capacity. The problem is the limited and costly available spectra to wireless data carriers. It has its slam not only on smart phones, but also on all wireless devices. In order to tackle the problem of spectrum underutilization, the concept of Cognitive Radio (CR) was proposed by Joseph Mitola III [1].The cognitive radio, built on a software-defined radio, is defined as an intelligent wireless communication system that is familiar of its environment and uses the technique of grasping by-building to learn from the environment and adapt to statistical variations in the input stimuli, with two primary objectives in mind highly dependable communication whenever and wherever needed well organized utilization of the radio spectrum [2].

Cognitive radio promises a low-cost, highly flexible alternative to the single-protocol wireless device, classic single-frequency band. By sensing and adjusting to its environment, such a device is able to fill voids in the wireless spectrum and can dramatically increase spectral efficiency [3]. Main concern of cognitive radio is to make sure that cognitive user will not inhibit with the licensed user while communicating in licensed spectrum. Based on available network information and other directives there are different undertaking by which secondary user access spectrum without interfering with primary user. These approaches include under lay, overlay and interweave paradigm [3] [4].

Interweaver paradigm uses opportunistic spectrum access method that was primary idea of cognitive radio. It is based on the fact of less utilization of spectrum which indicated that there are short term space-time frequency holes that could be utilized by cognitive users. Existence of these holes depends on time and geographical location. For systematic and interference free communication cognitive user needs activity based information of licensed and unlicensed users [5]. In more general perspective interweaver cognitive radios are smart systems that detect the unused spectrum opportunistically, utilizing it for communication and leaving the spectrum when primary user is detected thus avoiding considerable interference [6].

For this purpose the IEEE 802.22 Standard was launched. In this standard it is defined that Cognitive Radio based Regional Area Networks (RAN) in TV White Spaces (TVWS) [7]. Base stations (BS) and Customer Premise Equipments (CPEs) do spectrum sensing both in-band (used channels) and out-of band (channels not used). Out-of-band sensing done when CPE is idle. Spectrum Sensing is Quiet period and two phase sensing [8]. Fast sensing is some quiet time allocated in a frame. Fine sensing with several ms in occupied channels to look for transmitter info is Quiet period of multiple frames. Dynamic Frequency Hopping (DFH) Sensing is completed in parallel (Optional) [9].Spectrum manager and MAC 
communicate using interface B1. Management can configure spectrum manager and set policies using interface B2 Security layer authenticates senders of beacons to each other. Frame Control Header (FCH) is Maps' lengths, modulation and coding, usable subcarriers first downstream burst contains information about the frame. Downlink Map is burst lengths for this frame [10]. Upstream Map is Burst lengths for transmission from each CPE for next frame. Maps can be compressed Downstream Channel Descriptor (DCD) is PHY characteristics of downstream bursts (modulation, coding) Upstream Channel Descriptor (UCD) 0[11].

There are various tools available for the implementation of cognitive radios that are needed for the evaluation of the performance for the effective utilization of IEEE 802.22 for example NS2, Omnet++, Ansys, NETSIM etc [12]. Among all of them NETSIM is the best tool for the implementation of scenarios. It is very user friendly software as only drag and drop approach is followed. Some of the work is reported in previous years in this context. Beyond a simple review of scenarios by considering the viewpoints of several key players in wireless communication research and applications: regulators, standardization bodies, researchers from the engineering and economic/business communities, industrial partners and companies are investigated [13]. In that framework, two key issues related to scenario definition are addressed: An analysis of players that determine the evolution of scenarios, including both technical and economic/business aspects, Study of approaches for classification of CR deployment scenarios, with the aim of identifying a set of elements that allow creating taxonomy capable of fitting existing and new scenarios relevant to CR and SDR[12].

Now that all work are proposed theoretically so it is required to implement all the work practically using a simulation tool. Now we are implementing these scenarios using NETSIM. These Scenarios are on the basis of Scalability, no. of incumbents, distance, density i.e. no. of CPEs.

\section{BACKGROUND}

In order to understand the simulation work carried out for the cognitive radio networks based on IEEE 802.22 standards, the brief description of the perquisites has been enumerated below:

\section{IEEE 802.22 Standard :-}

IEEE 802.22, is a standard for wireless regional area network (WRAN) using white spaces in the television (TV) frequency spectrum. The development of the IEEE 802.22 WRAN standard is focused at using cognitive radio (CR) technology to allow sharing of geographically unused spectrum allocated to the television broadcast service, on a non-interfering basis, to bring broadband access to hard-to-reach, low population density areas, typical of rural environments, and is therefore timely and has the potential for a wide applicability worldwide. It is the first worldwide effort to explain a standardized air interface based on CR techniques for the opportunistic use of TV bands on a non-interfering basis [14].

IEEE 802.22 WRANs are designed to operate in the TV broadcast bands while assuring that no harmful interference is caused to the incumbent operation: digital TV and analog TV broadcasting, and low power licensed devices such as wireless microphones. The standard was expected to be finalized in Q1 2010, but was finally published in July 2011[15].
The concept of Spectrum Availability involves Spectrum Sensing used to ensure spectrum availability for primary users. Various types of signal specific and feature based sensing algorithms have been adjoined into the standard. The Standard recommends sensing algorithms to determine the signal type (Signal Classification). In Collaborative Sensing the group in general thinks that collaborative sensing will be useful. FCC $\mathrm{R} \& \mathrm{O}$ requires 'OR' rule based collaborative sensing. In its Correlation with Geo-location information it is stated that it is closely tied to collaborative sensing. It tries to cross check the spectral footprint of the detected signal based on location of the sensor. In Its Spectrum Access Authorization the BS is capable of de-authorizing a subscriber at any time. Sensing and incumbent database service used for spectrum access authorization[14]. The Spectrum Manager (SM) is capable of prohibiting a subscriber from registering if it does not have adequate sensing capabilities.

\section{Application Traffic Types: \\ Voice}

Codec is the component of any voice system that translates between analog speech and the bits used to transmit them. Every codec transmits a burst of data in a packet that can be reconstructed into voice. Five different standards of voice codec's are given which can be selected depending on the variations required [16].

Codec types:

\section{G.711}

G.711 is an ITU-T standard for audio companding. It is primarily used in telephony. The standard was released for usage in 1972. Its formal name is Pulse code modulation (PCM) of voice frequencies. it is mandatory standard in many technologies, for example in H.320 and H.323 specifications.. G.711, also known as Pulse Code Modulation (PCM), is a very commonly used waveform codec [17]. G.711 is a narrowband audio codec that provides toll-quality audio at $64 \mathrm{kbit} / \mathrm{s}$. it samples them at the rate of 8,000 samples per second and ravine audio signals in the range of $300-3400 \mathrm{~Hz}$, with the tolerance on that rate of 50 parts per million (ppm). Sampling frequency is $8 \mathrm{kHz}$. $64 \mathrm{kbit} / \mathrm{s}$ bitrate $(8 \mathrm{kHz}$ sampling frequency $\times 8$ bits per sample).G.711 is a waveform speech coder.

\section{G.723}

it is an ITU-T standard voice codec utilizing extensions of G.721 giving voice quality covering $300 \mathrm{~Hz}$ to $3400 \mathrm{~Hz}$ using Adaptive Differential Pulse Code Modulation (ADPCM) to 24 and $40 \mathrm{kbit} / \mathrm{s}$ for digital circuit multiplication equipment (DCME) applications [18]. The standard G.723 is obsolete and has been superseded by G.726.

\section{G.729}

G.729 is an audio data compression algorithm that compresses digital voice in packets of 10 milliseconds duration. It is officially described as Coding of speech at $8 \mathrm{kbit} / \mathrm{s}$ using code-excited linear prediction speech coding (CSACELP). Standard G.729 operates at a bit rate of $8 \mathrm{kbit} / \mathrm{s}$, but extensions gives rates of $6.4 \mathrm{kbit} / \mathrm{s}$ (Annex D, F, H, I, C+) and $11.8 \mathrm{kbit} / \mathrm{s}$ (Annex E, G, H, I, C+) for good and bad speech quality, consequently. Sampling frequency is $8 \mathrm{kHz} / 16$-bit (80 samples for $10 \mathrm{~ms}$ frames).Fixed bit rate (8 kbit/s $10 \mathrm{~ms}$ frames).Fixed frame size (10 bytes for $10 \mathrm{~ms}$ frame) [19]. 


\section{GSM-FR}

GSM-FR and FR stands for Full Rate (FR or GSMFR or GSM 06.10 or sometimes simply GSM) was the first and foremost digital speech coding standard used in the GSM digital mobile phone system. Codec's bit rate is 1.625 bits/audio sample (often padded out to 33 bytes/20 ms or $13.2 \mathrm{kbit} / \mathrm{s}$ ) or $13 \mathrm{kbit} / \mathrm{s}$. The quality of the coded speech is quite bad in terms of modern standards, but at the time of development, it was a good compromise between computational complexity and quality, needs only on the order of a million additions and multiplications per second. The codec is still widely used in networks around the world [20].

\section{GSM-EFR}

Enhanced Full Rate or EFR or GSM-EFR or GSM 06.60 is a speech coding standard that was developed in order to enhance the poor quality of GSM-Full Rate (FR) codec. Working at $12.2 \mathrm{kbit} / \mathrm{s}$ the EFR provides good quality in any noise free and background noise conditions. The EFR 12.2 kbit/s speech coding standard is compatible with the topmost AMR mode (both are ACELP) [21]. As the EFR helps to refine call quality, this codec has higher computational complexity, which in a mobile device can potentially result in an increase in energy consumption as high as 5\% compared to 'old' FR codec.

In Packet Size Distribution the options available for distribution are constant and Exponential. Packet Size (Bytes) sets the size of the packets being generated by the chosen distribution. By default 1460 bytes is entered. Inter Arrival Time indicates the time gap between packets. In Distribution options available for distribution are Constant or Exponential [22].

Inter Arrival Time enter the average inter-arrival time between packets. A lower inter-arrival time would lead to a higher creation rate and the vice versa. By default 20000 Micro Sec is entered.

Video is an electronic medium for the recording, copying and broadcasting of moving visual images.

\section{Model Type-}

Continuous Normal VBR is the simplest of all models. It uses Normal Distribution for the creation of bits per pixel. In this model, consecutive packet sizes are independent of each other [23].

Continuous State Autoregressive Markov incorporates the autocorrelation between the frames. Also, current packet size depends on the previous packet size via the first order autoregressive Markov process [24]. In Quantized State Continuous Time Markov the bit rate is quantized into finite discreet levels. This model takes uniform quantization step as A bits/pixel. There are $M+1$ possible levels $(0, A, \ldots .$. , MA).Transitions between levels are assumed to occur with exponential rates that may depend on the current level [25]. This model is approximating the bit rate by a continuous time process $\lambda(\mathrm{t})$ with discreet jumps at random Poisson time.

In Simple IPB Composite Model, the frames are organized as IBВРВВРВВРВВIВВРВВ... i.e., 12 frames in a Group of Pictures(GOP).Generate $\mathrm{X} 0$ from a Gaussian distribution $\mathrm{N}(0$, y 0).Set initial value $\mathrm{N} 0=0, \mathrm{D} 0=1 \mathrm{H}$ is called the Hurst parameter $\mathrm{k}-\beta$ is used as the ACF of a self-similar process. We get the value of $\mathrm{H}$ parameter for a self-similar process using the relationship [26]. NetSim provides distinct quantitative metrics at various abstraction levels such as Network Metrics, Link Metrics, TCP Metrics, Application Metrics, etc at the end of simulation. With the help of metrics, users can analyze the behavior of the modeled network and can compare the impact of different algorithms on end-to-end behavior[27].

\section{Performance parameters:}

In Order to quantify the performance of the CRNs The Important parameters used in this work are mentioned below:

In Network metrics users can view the values of the metrics obtained based on the overall network. Link Metrics displays the values of the metrics pertaining to each link. Error Packets are total number of packets error in the link inclusive of data and control packets [28]. Payload Transmitted is the total payload transmitted in the link. Throughput is the total user data (or) payload delivered to their respective destination every second.

$$
\begin{gathered}
\text { Calculation: } \\
\text { Throughput }(\text { in Mbps })=\frac{\text { Total payload delivered to destination (bytes) } * 8}{\text { Simulation Time }(\text { Micro sec) }}
\end{gathered}
$$

Delay is the average amount of time taken calculated for all the packets to reach the destination from the source.

\section{Common properties for all the traffic types :}

Application ID represents the unique identification number of the application.

Start time represents the start time of the application in seconds.

End time represent the end time of the application in seconds. Suppose Start time is 1 and end time is 10 then application starts generating traffic at 1st second and ends at 10th second. Source ID represents the unique identification number of the source.

Destination ID represents the unique identification numbers of the destination.

\section{III . SIMULATION METHODOLOGY AND ENVOIRMENT}

To find out the behavioral characteristics of the cognitive radio networks using voice and video traffic types the simulations have been done. The methodology of the simulator used has been described in this section. We have to create new experiment by selecting the technology as cognitive radio technology after that the work window will open in which we will create a scenario by using various network devices such as CPEs, routers, Incumbents etc. Then to trace all the packets in the transmission we need to enable packet tracer that is wire shark in the source node. We have to switch it on by applying its status as online. After setting these properties we need to select the performance metrics upon which we have to evaluate the results. After that application traffic type should be defined for each and every node. After completing all these steps we need to set the simulation time and run the simulation to get the required results. After the simulation is completed we get the metrics file for every application in which we will get all the required readings and results.

Now the flow chart is given below : 


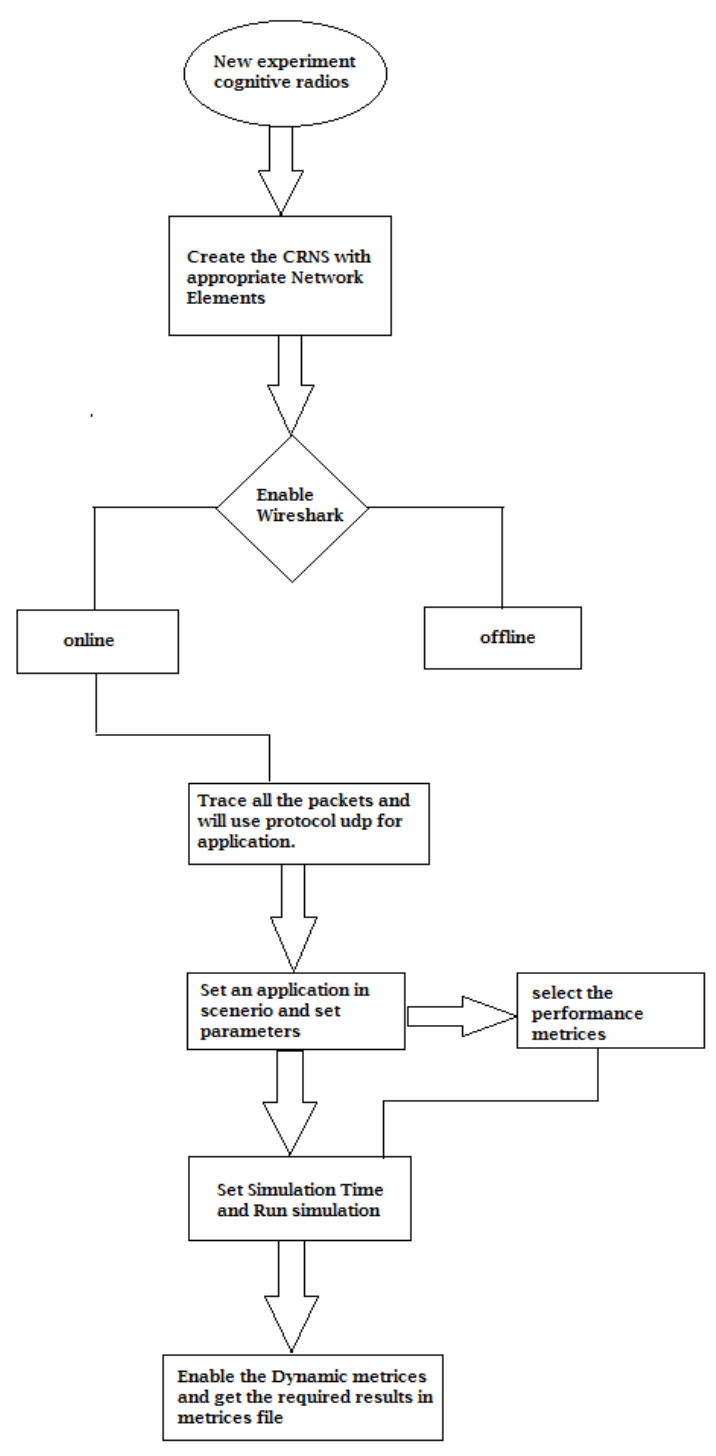

Chart I: Showing the Step by Step procedure of simulation.

\section{ENVIORMENT}

To perform the Simulation we not only need to set the scenario but also need to set the parameters according to which the results are calculated and these parameters are:

Table I: Showing the Parameters of wired node in the network.

\begin{tabular}{lc}
\hline & Global Properties \\
\hline Device Type & Wired node \\
Maximum Segment Size & online \\
Congestion Control Algorithm & Transport Layer \\
Window Size & OLD_TAHOE \\
Protocol & 8 \\
Segment Size & Udp \\
& 1460 \\
Protocol & Network Layer \\
ICPM_cont_polling & IPv4 \\
& 3 \\
& Interface Ethernet \\
IP_Address & Network Layer \\
Subnet_Mask & 11.1 .1 .2 \\
Protocol & 255.255.0.0 \\
ARP_Retry_Interval & ARP \\
\hline Protocol & 10 \\
MAC_adderss & Data Link Layer \\
\hline
\end{tabular}

Table II: Showing the properties of router in the scenario.

\begin{tabular}{|c|c|}
\hline \multicolumn{2}{|c|}{ Global Properties } \\
\hline Device Type & Router \\
\hline Wireshark & Disable \\
\hline \multicolumn{2}{|c|}{ Transport Layer } \\
\hline Maximum Segment Size & 1460 \\
\hline Congestion Control Algorithm & OLD_TAHOE \\
\hline Window Size & $8-2$ \\
\hline Protocol & Udp \\
\hline Segment Size & 1472 \\
\hline \multicolumn{2}{|c|}{ Network Layer } \\
\hline Protocol & IPv4 \\
\hline ICPM_cont_polling & 3 \\
\hline Firewall_Staus & False \\
\hline \multicolumn{2}{|c|}{ Interface Ethernet } \\
\hline \multicolumn{2}{|c|}{ Network Layer } \\
\hline IP_Address & 11.1 .1 .2 \\
\hline Subnet Mask & 255.255 .0 .0 \\
\hline Buffer size(MB) & 8 \\
\hline Scheduling Type & FIFO \\
\hline Protocol & ARP \\
\hline ARP_Retry_Interval & 10 \\
\hline \multicolumn{2}{|c|}{ Data Link Layer } \\
\hline Protocol & Ethernet \\
\hline MAC_adderss & 2B3B15691E09 \\
\hline
\end{tabular}

Table III: Showing the properties of Base Station in the scenario.

\begin{tabular}{lc}
\hline & Global Properties \\
\hline $\begin{array}{l}\text { Device Type } \\
\text { Wireshark }\end{array}$ & $\begin{array}{c}\text { Base Station } \\
\text { offline }\end{array}$ \\
\hline Protocol & Data Link Layer \\
\hline Duplexing & IEEE 802.22 \\
\hline Sensing period & Time division multiplexing \\
Sensing_Interval & 16 (symbols) \\
\hline Channel check time & 200 (Frames) \\
& 30 \\
\hline Min frequency & Physical Layer \\
Max Frequency & 54 \\
\hline Channel Bandwidth & 60 \\
Modulation & 6 \\
Coding rate & 64 Qam \\
Multipe_Access & $2 \backslash 3$ \\
\hline CPFactor & OFDMA \\
DL_UL ratio & $1 / 16$ \\
Connection medium & $1: 2$ \\
& Wireless \\
\hline
\end{tabular}

Table IV: Showing the properties of the incumbent in the scenario.

\begin{tabular}{cc}
\multicolumn{2}{c}{ Operation Properties } \\
\hline Oper_frequency_start & 54 \\
Oper_frequecy_end & 60 \\
Oper_Distribution & Exponential \\
\hline
\end{tabular}

Table V: Showing the properties of a CPE in the scenario.

\begin{tabular}{|c|c|}
\hline \multicolumn{2}{|c|}{ Global Properties } \\
\hline Device Type & $\mathrm{CPE}$ \\
\hline Wireshark & offline \\
\hline \multicolumn{2}{|c|}{ Transport Layer } \\
\hline Maximum Segment Size & 1460 \\
\hline Congestion Control Algorithm & OLD_TAHOE \\
\hline Window SIze & $8--$ \\
\hline Protocol & $\mathrm{Udp}$ \\
\hline Segment Size & 1472 \\
\hline \multicolumn{2}{|c|}{ Network Layer } \\
\hline Protocol & IPv4 \\
\hline ICPM_cont_polling & 3 \\
\hline \multicolumn{2}{|c|}{ Interface Ethernet } \\
\hline \multicolumn{2}{|c|}{ Network Layer } \\
\hline IP_Address & 11.1.1.2 \\
\hline Subnet Mask & 255.255 .0 .0 \\
\hline Protocol & ARP \\
\hline ARP_Retry_Interval & 10 \\
\hline \multicolumn{2}{|c|}{ Data Link Layer } \\
\hline Protocol & Ethernet \\
\hline MAC_adderss & D0567F0898DD \\
\hline
\end{tabular}


Table VI: Showing Application Properties including various codec and various model types.

\begin{tabular}{ll}
\hline & Application Properties \\
\hline Application Type & Video,voice \\
Source_Id & Wired Node \\
Destination_Id & CPES \\
Start Time & Os \\
End Time & 100 s \\
Codec & G.711,G.723,G.729,GSM-FR,GSM-EFR \\
Model type & CN-VBR,QTM, \\
& CSAM, Simple_Ipb_Composite_Model \\
\hline
\end{tabular}

The Scenario on which we are working is :

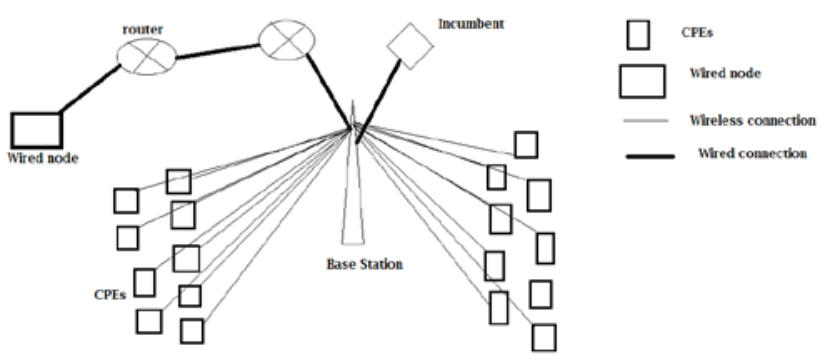

Fig I Showing scenario with 20 nodes using CRNs.

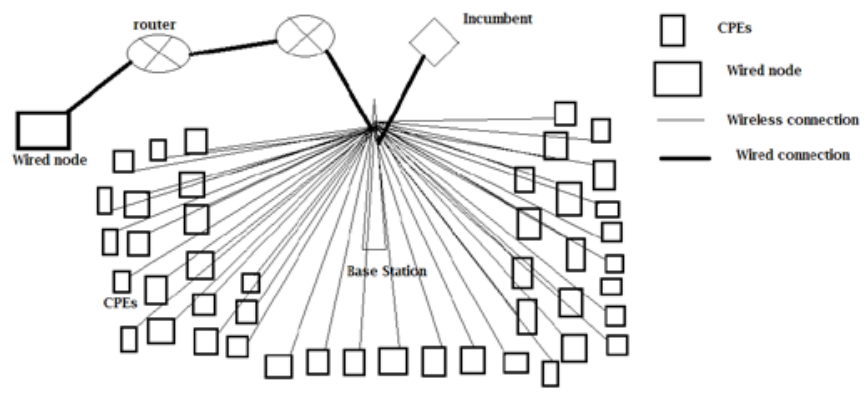

FIG II: Showing the scenario with 50 nodes to increase the load on the base station.

\section{SIMULATION RESULTS AND DISCUSSIONS}

As per the scenario and the parameters set in previous section the result table is obtained as follows:

Table I: Showing throughput of various codec in voice application

\begin{tabular}{|l|l|l|l|l|l|l|l|l|}
\hline $\begin{array}{l}\text { Experime } \\
\text { nt }\end{array}$ & $\begin{array}{l}\text { Applicati } \\
\text { on }\end{array}$ & codec & $\begin{array}{l}\text { Distan } \\
\text { ce }\end{array}$ & $\begin{array}{l}\text { Densit } \\
\text { y }\end{array}$ & $\begin{array}{l}\text { Incumbe } \\
\text { nts }\end{array}$ & $\begin{array}{l}\text { Max } \\
\text { throughput }\end{array}$ & $\begin{array}{l}\text { Min } \\
\text { throughput }\end{array}$ & $\begin{array}{l}\text { Avg } \\
\text { Through } \\
\text { put(mbps } \\
\text { (mb }\end{array}$ \\
\hline 1. & voice & G.711 & $10 \mathrm{~km}$ & 20 & 5 & 0.025600 & 0.025149 & 0.025344 \\
\hline 2. & voice & G.723 & $10 \mathrm{~km}$ & 20 & 5 & 0.006374 & 0.006123 & 0.006212 \\
\hline 3. & voice & G.729 & $10 \mathrm{~km}$ & 20 & 5 & 0.007904 & 0.007827 & 0.007852 \\
\hline 4. & voice & $\begin{array}{l}\text { GSM- } \\
\text { FR }\end{array}$ & $10 \mathrm{~km}$ & 20 & 5 & 0.012778 & 0.012672 & 0.012523 \\
\hline 5. & Voice & $\begin{array}{l}\text { GSM- } \\
\text { EFR }\end{array}$ & $10 \mathrm{~km}$ & 20 & 5 & 0.012003 & 0.011904 & 0.011991 \\
\hline 6. & voice & G.711 & $10 \mathrm{~km}$ & 50 & 5 & 0.006656 & 0.006400 & 0.006548 \\
\hline 7. & voice & G.729 & $10 \mathrm{~km}$ & 50 & 5 & 0.003680 & 0.003616 & 0.003645 \\
\hline 8. & voice & G.723 & $10 \mathrm{~km}$ & 50 & 5 & 0.004416 & 0.004215 & 0.004325 \\
\hline 9. & voice & $\begin{array}{l}\text { GSM- } \\
\text { FR }\end{array}$ & $10 \mathrm{~km}$ & 50 & 5 & 0.009765 & 0.009425 & 0.009621 \\
\hline 10. & voice & GSM- & $10 \mathrm{~km}$ & 50 & 5 & 0.0090023 & 0.008902 & 0.008991 \\
\hline
\end{tabular}

In the above table we can see that the scenario we have chosen is giving us the readings for maximum and minimum throughput and form all the reading that we got from each node in the scenario we have calculated the average throughput of whole scenario.

Table II : Showing Delay and PE:PT in various codecs in CNR.

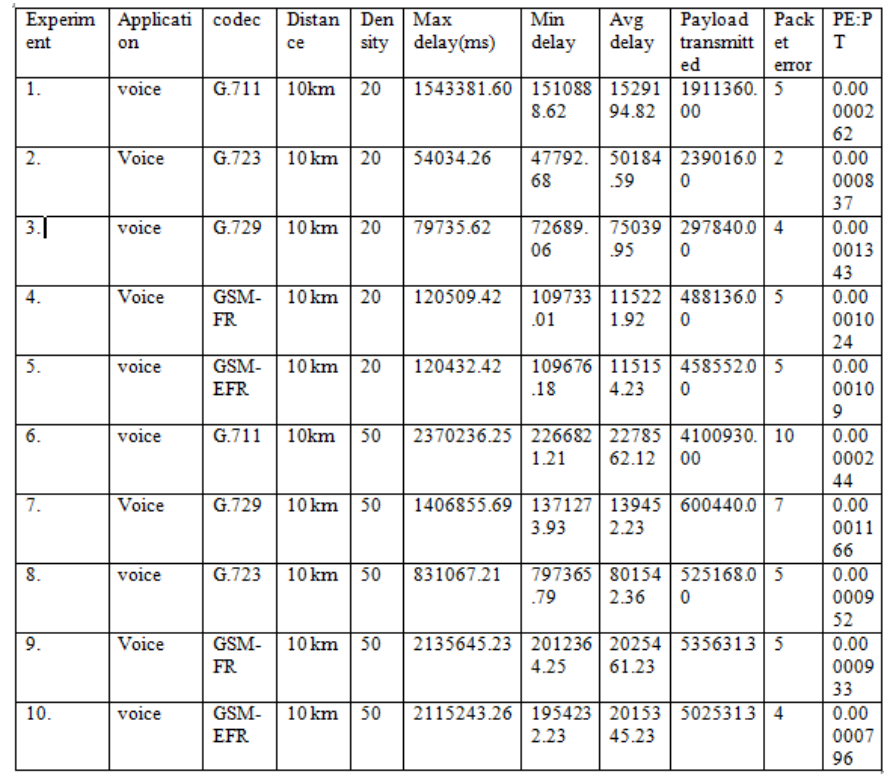

In the above table we can see that the readings we are getting from the scenario in the form of maximum, minimum and average delay and we can see that how it is increasing with the distance and the density From the above table we can also see that the payload transmitted and the respective packet error in the particular scenario and how it is varying with density and distance.

Table III: showing maximum, minimum and average throughput of various video model types of various scenario.

\begin{tabular}{|l|l|l|l|l|l|l|l|l|}
\hline $\begin{array}{l}\text { Expe } \\
\text { nime } \\
\text { nt }\end{array}$ & $\begin{array}{l}\text { Applica } \\
\text { tion }\end{array}$ & Model type & $\begin{array}{l}\text { Distan } \\
\text { ce }\end{array}$ & $\begin{array}{l}\text { Densit } \\
\text { y }\end{array}$ & $\begin{array}{l}\text { Incumbe } \\
\text { nts }\end{array}$ & $\begin{array}{l}\text { Max } \\
\text { throughput }\end{array}$ & $\begin{array}{l}\text { Min } \\
\text { throughput }\end{array}$ & $\begin{array}{l}\text { Avg } \\
\text { Throughp } \\
\text { ut(mbps) }\end{array}$ \\
\hline 1 & Video & $\begin{array}{l}\text { Cont. } \\
\text { Normal } \\
\text { vbr }\end{array}$ & $10 \mathrm{~km}$ & 20 & 5 & 0.030208 & 0.024688 & 0.026150 \\
\hline 2. & video & $\begin{array}{l}\text { Cont. } \\
\text { State } \\
\text { Autorefressiv } \\
\text { eMarkov }\end{array}$ & $10 \mathrm{~km}$ & 20 & 5 & 0.007824 & 0.006125 & 0.006523 \\
\hline 3. & video & $\begin{array}{l}\text { Quantised } \\
\text { state cont. } \\
\text { Timemarkov }\end{array}$ & $10 \mathrm{~km}$ & 20 & 5 & 0.021917 & 0.015363 & 0.018643 \\
\hline 4. & video & $\begin{array}{l}\text { Simple ipb } \\
\text { composite } \\
\text { model }\end{array}$ & $10 \mathrm{~km}$ & 20 & 5 & 0.036198 & 0.023158 & 0.027190 \\
\hline 5. & Video & $\begin{array}{l}\text { Cont. } \\
\text { Normal } \\
\text { vbr }\end{array}$ & $10 \mathrm{~km}$ & 50 & 5 & 0.009134 & 0.005549 & 0.007425 \\
\hline 6. & video & $\begin{array}{l}\text { Cont. } \\
\text { State } \\
\text { Autorefressiv } \\
\text { eMarkov }\end{array}$ & $10 \mathrm{~km}$ & 50 & 5 & 0.004025 & 0.003012 & 0.003154 \\
\hline 7. & video & $\begin{array}{l}\text { Quantised } \\
\text { state cont. } \\
\text { Timemarkov }\end{array}$ & $10 \mathrm{~km}$ & 50 & 5 & 0.005681 & 0.004521 & 0.005012 \\
\hline 8. & video & $\begin{array}{l}\text { Simple ipb } \\
\text { composite } \\
\text { model }\end{array}$ & $10 \mathrm{~km}$ & 50 & 5 & 0.008501 & 0.006510 & 0.007524 \\
\hline
\end{tabular}


Table IV: Showing the maximum, minimum and average delay

In various model type in the scenario.

\begin{tabular}{|l|l|l|l|l|l|l|l|}
\hline $\begin{array}{l}\text { Expe } \\
\text { ime } \\
\text { nt }\end{array}$ & $\begin{array}{l}\text { Applicat } \\
\text { ion }\end{array}$ & Model type & $\begin{array}{l}\text { Dista } \\
\text { nce }\end{array}$ & $\begin{array}{l}\text { Dens } \\
\text { ity }\end{array}$ & $\begin{array}{l}\text { Max } \\
\text { delay(ms) }\end{array}$ & Min delay & Avg delay \\
\hline 1. & Video & CN-VBR & $10 \mathrm{~km}$ & 20 & 1296732.51 & 981089.67 & 962510.02 \\
\hline 2. & video & CSAM & $10 \mathrm{~km}$ & 20 & 734101.712 & 410741.318 & 512881.32 \\
\hline 3. & video & QTM & $10 \mathrm{~km}$ & 20 & 677393.42 & 533302.47 & 639581.23 \\
\hline 4. & video & SICM & $10 \mathrm{~m}$ & 20 & 1074113.43 & 818537.12 & 952128.69 \\
\hline 5. & video & CN-VBR & $10 \mathrm{~km}$ & 50 & 2849148.72 & 162982.90 & 1952431.23 \\
\hline 6. & video & CSAM & $10 \mathrm{~km}$ & 50 & 2215431.36 & 2012541.23 & 2145642.85 \\
\hline 7. & Video & QTM & $10 \mathrm{~km}$ & 50 & 2564135.23 & 2451356.23 & 2495246.89 \\
\hline 8. & Video & SICM & $10 \mathrm{~km}$ & 50 & 2641545.23 & 2458762.12 & 2546153.67 \\
\hline
\end{tabular}

Table V: Showing the payload Transmitted, Packet error and the ratio between them.

\begin{tabular}{|l|l|l|l|l|l|l|l|}
\hline $\begin{array}{l}\text { Expe } \\
\text { rime } \\
\text { nt }\end{array}$ & $\begin{array}{l}\text { Applic } \\
\text { ation }\end{array}$ & $\begin{array}{l}\text { Model } \\
\text { type }\end{array}$ & $\begin{array}{l}\text { Dista } \\
\text { nce }\end{array}$ & Density & $\begin{array}{l}\text { Payload } \\
\text { transmitted }\end{array}$ & Packet error & PE:PT \\
\hline 1. & Video & CN-VBR & $10 \mathrm{~km}$ & 20 & 1616002.00 & 4 & 0.00000248 \\
\hline 2. & video & CSAM & $10 \mathrm{~km}$ & 20 & 354837.95 & 1 & 0.00000282 \\
\hline 3. & video & QTM & $10 \mathrm{~km}$ & 20 & 1470539.00 & 4 & 0.00000272 \\
\hline 4. & video & SICM & $10 \mathrm{~m}$ & 20 & 3847357.00 & 6 & 0.00000156 \\
\hline 5. & video & CN-VBR & $10 \mathrm{~km}$ & 50 & 3242049.00 & 5 & 0.00000154 \\
\hline 6. & video & CSAM & $10 \mathrm{~km}$ & 50 & 2546311.00 & 2 & 0.00000079 \\
\hline 7. & Video & QTM & $10 \mathrm{~km}$ & 50 & 2145632.00 & 7 & 0.00000326 \\
\hline 8. & Video & SICM & $10 \mathrm{~km}$ & 50 & 2013517.00 & 5 & 0.00000248 \\
\hline
\end{tabular}

\section{Graphs:}

In this section we have drawn various graphs for the data representation as well as the data interpretation. The graphs are drawn as follows:

Graphs for the video application:

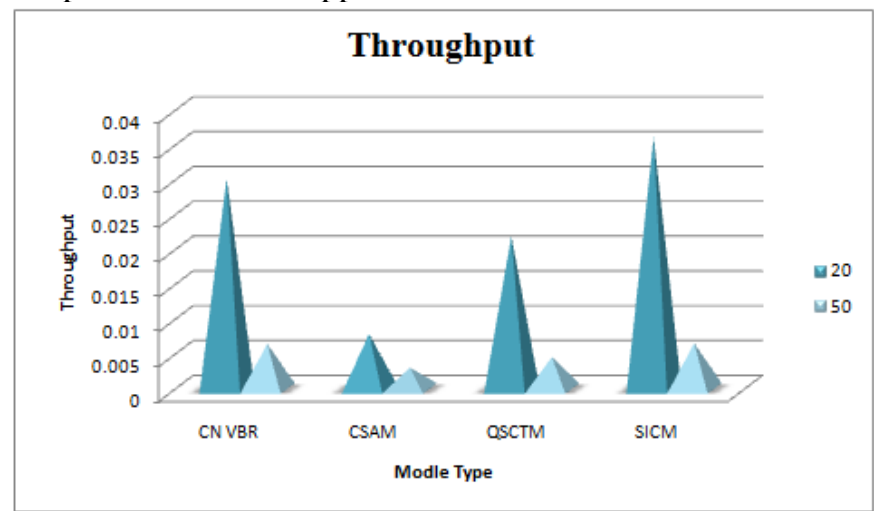

Graph I: Showing the comparison of average throughput in various model types of video application.

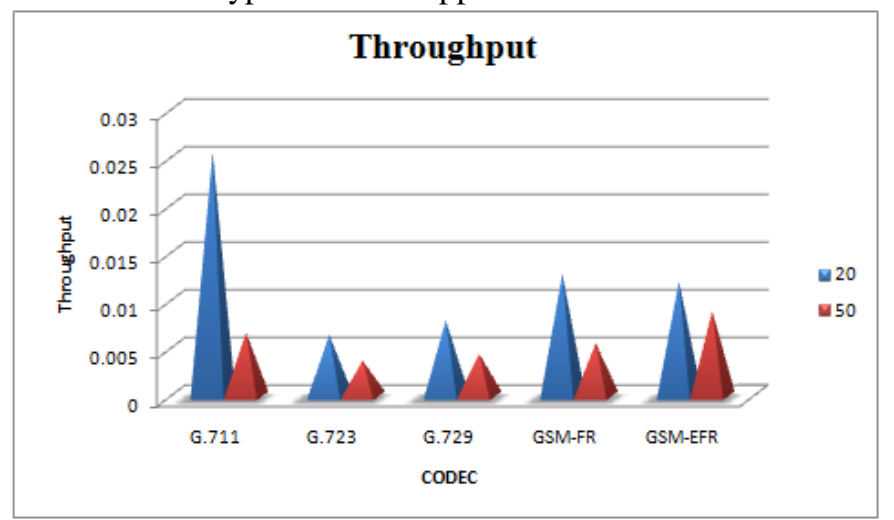

Graph II: Showing the comparison of throughput of various codec.

In the first graph we can see the comparison of throughput in different model types in video application. In this application we got four model types that are continuous normal VBR, Cont. State Autorefressive Markov, Quantized state cont.
Time markov, Simple ipb composite model and among all of them we can see that Simple ipb composite model is giving us the best results in terms of throughput as compared to other models in that application. In the second graph we can see the comparison of throughput in different codecs in voice application. In this application we got five codecs G.711, G.723, G.729, GSM-FR,GSM-EFR and among all of them we can see that G.711is giving us the best results in terms of throughput as compared to other codec in that application.

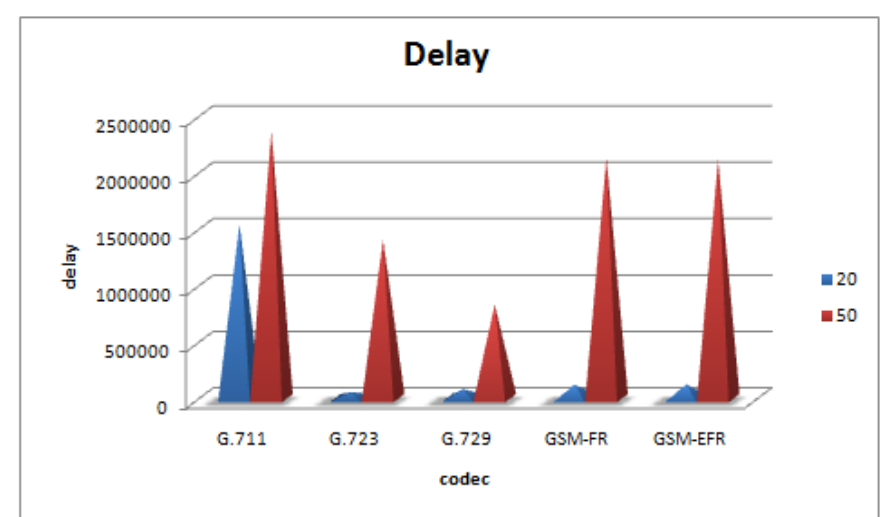

Graph II: Showing the comparison of average Delay in various types of codec in voice application.

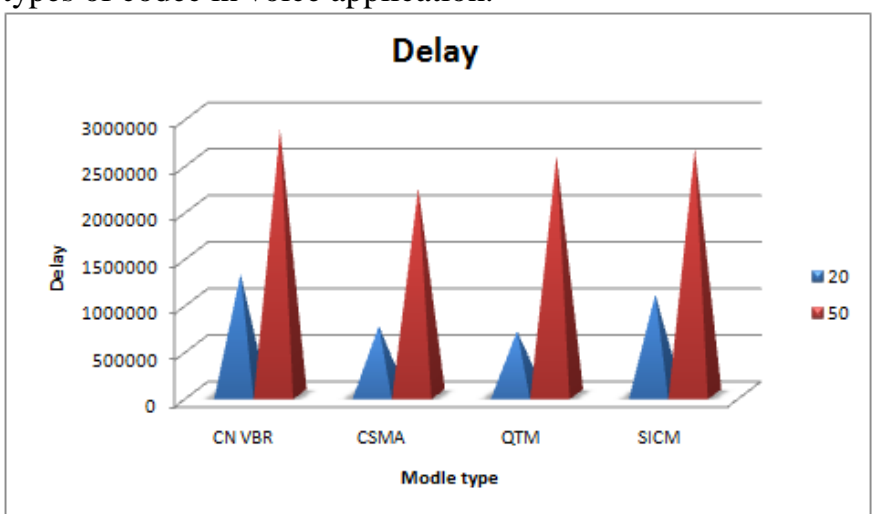

Graph III: Showing the Comparison of delay in various model types of video application.

In the first graph we can see the comparison of delays in different model types in video application. In this application we got four model types that are continuous normal VBR, Cont. State Autorefressive Markov, Quantized state cont. Time markov, Simple ipb composite model and among all of them we can see that Simple ipb composite model is giving us the best results in terms of delay as compared to other models in that application. In the second graph we can see the comparison of delay in different codecs in voice application. In this application we got five codecs G.711, G.723, G.729, GSM-FR,GSM-EFR and among all of them we can see that G.711is giving us the best results in terms of delay as compared to other codecs in that application. 


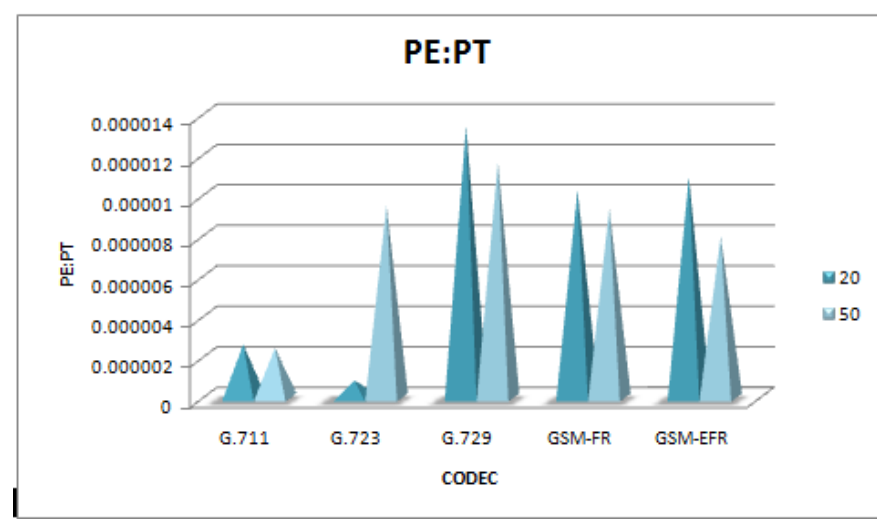

Graph III: Showing the comparison of the ratio of Payload transmitted to the packet error in various model types of video application.

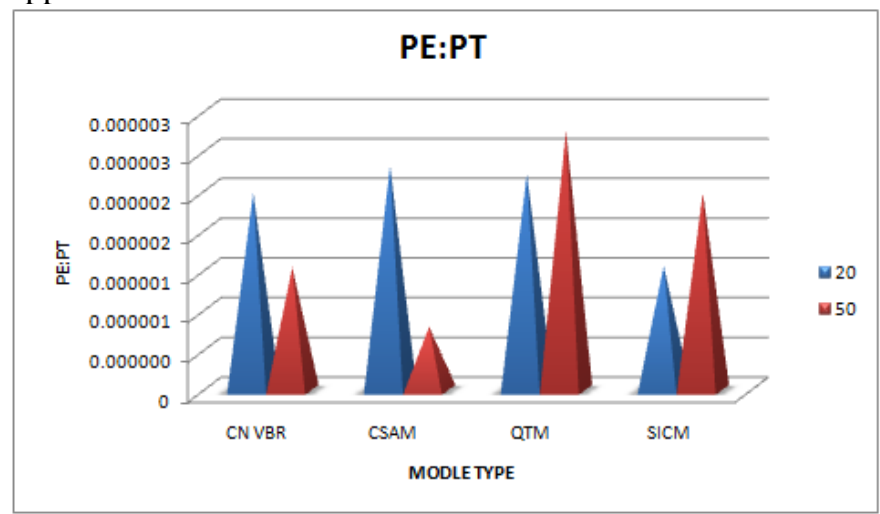

Graph III: Showing the comparison of the ratio of Payload transmitted to the packet error in various codecs in voice application.

In the first graph we can see the comparison of PE:PT in different model types in video application. In this application we got four model types that are continuous normal VBR, Cont. State Autorefressive Markov, Quantized state cont. Time markov, Simple ipb composite model and among all of them we can see that Simple ipb composite model is giving us the best results in terms of PE:PT as compared to other models in that application. In the second graph we can see the comparison of PE:PT in different codecs in voice application. In this application we got five codecs G.711, G.723, G.729, GSM-FR,GSM-EFR and among all of them we can see that G.711is giving us the best results in terms of PE:PT as compared to other codecs in that application. Packet error is maximum in qtm model type as compared to payload transmitted.

From all of the above results we can conclude that the among all the model types and codecs in video and voice application the best model type is Simple ipb composite model and best codec is G.711. in terms in throughput, delay and PE:PT parameters. For efficient performance of scenario these model type and codec should be used.

\section{CONCLUSION}

The ever-increasing pace of technology advancement and the related increases in the demands for the various applications the technology has enabled have rendered this "studied opinion"-based allocation scheme virtually impossible to manage in the developed countries of the world [29].
Cognitive radio, which is one of the efforts to utilize the obtainable spectrum more efficiently through opportunistic spectrum usage, has become a stimulating and promising concept. One of the essential elements of cognitive radio is sensing the obtainable spectrum opportunities. In this paper we took scenario various traffic types such as video and voice and checked that how the overall throughput and delay is varying with respect to codec and model type. From all of the above results we can conclude that the among all the model types and codec in video and voice application the best model type is Simple ipb composite model and best codec is G.711. in terms in throughput, delay and PE:PT parameters. For efficient performance of scenario these model type and codec should be used. As this technology has a huge scope in the future therefore the research work can help in making the maximum possible optimum use of it.

\section{REFERENCES}

[1] Joseph Mitola III and G. Q. Maguire, “Cognitive radio: making software radios more personal,” IEEE Personal Communications, vol. 6, no. 4, pp. 13-18, 1999.

[2] Simon Haykin, Life Fellow,," Cognitive Radio: BrainEmpowered wireless Communications”, IEEE JOURNAL ON SELECTED AREAS IN COMMUNICATIONS, vol. 23, no. 2, February 2005.

[3] Natasha Devroye, Patrick Mitran, Student Member, IEEE, and Vahid Tarokh," Achievable Rates in Cognitive Radio Channels", IEEE transaction on information theory, vol. 52, no. 5, MAY 2006.

[4] Kim, S.J. and G. B. Giannakis, 2009. Rate Optimal and Reduced-Complexity Sequential Sensing Algorithms for Cognitive OFDM Radios. In the proceedings of 2009 43rd annual conference on Information Science and Systems, pp: 141-146.

[5] Mitola, J., G.Q. Maguire, 1999. Cognitive Radio: Making Software Radios More Personal. J. of Personal Communication, IEEE., 6 (4): 13-18.

[6] Zhang, Q., G.J.M. Smit, L.T. Smit, A. Kokkeler, F.W. Hoeksema and M. Heskamp, 2005. A Reconfigurable Platform for Cognitive Radio. In the proceedings of 2005 2nd International Conference on Mobile Technology, Applications and Systems, pp: 1-5.

[7] Haykin, S., 2005. Cognitive Radio: Brain-Empowered Wireless Communication. IEEE Journal on Selected Areas in Communications. 23 (2): 201-220.

[8] Digham, F.F., 2008. Joint Power and Channel Allocation for Cognitive Radios. In the proceedings of 2008 Wireless Communications and Networking Conference, pp: 882 - 887.

[9] Alex, Z.C., Sivaraman and S.K. Vasudevan, 2010. Software Defined Radio Implementation (With simulation \& analysis).Intl. J. of Computer Applications., 4 (8): 21-27.

[10] Mitola, J., 2000. Software Defined Radio Architecture Evolution: Foundations, Technology Tradeoffs, and Architecture Implementations. IEICE Transactions on Communications, E83B (6):1165-1173.

[11] Zurutuza, N., 2012. Cognitive Radio, Fundamental Performance Analysis for Interweave Opportunistic Access Model. www.stanford.edu/ naroa/ee359project.pdf.

[12] Goldsmith, A., S.A. Jafar, I. Maric', and S. Srinivasa, 2009. Breaking Spectrum Gridlock with Cognitive Radios: An Information Theoretic Perspective. J. of the IEEE., 97 (5): $894-$ 914.

[13] Wang, B., and K. J. Ray Liu, 2011. Advances in Cognitive Radio Networks: A Survey. IEEE J. of Selected Topics in Signal Processing,. 5 (1): 5-23.

[14] Reed, J., C. Bostian, 2006. Understanding the Issues in Software Defined Cognitive Radio,” in Dyspan, Dublin, Ireland. 
[15] Shankar, S.N., 2007. Squeezing the Most Out of Cognitive Radio: A Joint MAC/PHY Perspective. In the proceedings of 2007 IEEE International Conference on Acoustics, Speech and Signal Processing, pp: 1361-1364.

[16] Kaustav Ghosh* Department of Computer Science, St. Xaviers College Autonomous (Kolkata), "India Security issues and challenges in cognitive radio network : a comprehensive study", ACCENTS Transactions on Information Security, Vol 1(1) ISSN (Online): 2455-7196

[17] J. Wang, M. Ghosh, and K. Challapali, "Emerging cognitive radio applications: a survey,” IEEE communication magazine, vol. 49, no. 3, pp. 74-81, 2011.

[18] Amna Saad Kamil, and Ibrahim Khider "Open Research issues in Cognitive Radio"16thTelecommunications Forum TELFOR, Serbia , Belgrade, 25-27 Nov., 2008.

[19] R.W. Brodersen, A. Wolisz, D. Cabric, S.M. Mishra, D. Willkomm, "Corvus: a cognitive radio approach for usage of virtual unlicensed spectrum”, Berkeley Wireless Research Center (BWRC) White paper, 2004.

[18] Eamonn O Nuallain, “A Proposed Propagation-based Methodology with which to address the Hidden Node Problem and Security/Reliability Issues in Cognitive Radio”, 4th International Conference on Wireless Communications, Networking and Mobile Computing, 2008. WiCOM '08.

[19] Raul Etkin, Abhay K. Parekh, David Tse, "Spectrum Sharing for Unlicensed Bands," IEEE Journal on Selected Areas in Communications, vol. 25, pp. 517-528, April 2007.

[20] http://www.hindawi.com/journals/vlsi/2012/716476/

[21] J. Arias, L. Quintanilla, J. Segundo, L. Enríquez, J. Vicente, and J. M. Hernández-Mangas, "Parallel continuous-time $\Delta \Sigma$ ADC for OFDM UWB receivers,” IEEE Transactions on Circuits and Systems I, vol. 56, no. 7, pp. 1478-1487, 2009.

[22] Federal Communications Commission, "Spectrum Policy Task Force”,Rep ET Docket no.02-135,Nov. 2002

[23] R. Tandra and A. Sahai, "SNR walls for signal detection," IEEE Journal on Selected Topics in Signal Processing, vol. 2, no. 1, pp. 4-17, 2008.

[24] Jagsir Singh, Roopali Garg \& Inderdeep Kaur Aulakh, "Effect of OFDM in Cognitive Radio: Advantages \& Issues”, Second International Conference on Computational Intelligence \& Communication Technology,2016

[25] Goutam Ghosh1 , Prasun Das2 and Subhajit Chatterjee3,” Cognitive Radio And Dynamic Spectrum Access - A Study”, International Journal of Next-Generation Networks (IJNGN) Vol.6, No.1, March 2014.

[26] Cheng-Xiang Wang, Fourat Haider, Xiqi Gao and Xiao-Hu You, Yang Yang, Dongfeng Yuan, Hadi M. Aggoune, Harald Haas, Simon Fletcher, Erol Hepsaydir," Cellular Architecture and Key Technologies for 5G Wireless Communication Networks”, IEEE Communications Magazine,2014

[27] William Krenik and Anuj Batra,” Cognitive Radio Techniques for Wide Area Networks", IEEE Communications Magazine,2014

[28] Hisham a. Mahmoud, Tevfik Yucek and Huseyin Arslan,” OFDM For Cognitive Radio: Merits and Challenges”, IEEE Wireless Communications, April 2009.

[29] Alexender M. Wyglinski, Maziar Nekovee, Thomas hou," Cognitive radio communication and network", ELSEVIER, April 2010. 